\title{
Network Sensor for Brain Wave with Automated Robot Assistance
}

\author{
Bhukya Krishna, K. Ranjith Reddy
}

\begin{abstract}
Human thoughts consists of of a massive variety of interconnected neurons. The examples of collaboration among those neurons are spoken to as issues and enthusiastic states. As indicated through way of the human contemplations, this example will change which thusly produce particular electric powered waves. A muscle compression will likewise create a one in every of a kind electrical sign. a variety of these electric powered waves will be detected by way of the cerebrum wave sensor and it'll change over the records into parcels and transmit thru Bluetooth medium.Level analyzer unit (LAU) gets the thoughts wave crude records and it's going to listen and device the signal using MATLAB level that's seemed in data getting ready unit. At that factor the manage directions may be transmitted to the robotic it truly is the assistive robotic. With this whole framework, we are able to drift a robotic as consistent with the imparting instructions to the robot and it very well can be have become by means of squint problems and it has an inclination to be grew to become by flicker muscle constriction.
\end{abstract}

Electroencephalography (EEG) is the estimation of electrical motion inside the residing mind. in this assignment we applied a brainwave sensor to dissect the EEG alerts. This plan speak about making geared up and recording the crude EEG signal from the thoughts Wave sensor within the MATLAB situation and through WIFI transmission control recommendations can be exceeded to the robotic section. thoughts wave sensors are not utilized in clinical use, however are applied inside the thoughts Brain Control Interface (BCI).

The BCI is a prompt correspondence pathway among the cerebrum and an outside framework to offer direct correspondence and control between the human personality and substantial gadgets through disentangling various instances of cerebrum movement into guidelines step by step. This endeavor works of art fuses of a Processor utilizing cerebrum wave sensor and arranged unit block prominence unit as device parts and a fruitful musings signal system utilizing Matlab organize. directly, the proprietor wants to test whether the automated move or never again. on the off hazard that he's a now not strolling, by then the automated will obviously start. Be that as it can, at the off risk that he's ordinary mode, by then the vehicle will run and there's no notice. while the vehicle got gleaming request it will prevent at any rate the spot. what's more, if the owner wants to move the car he has a need to come ordinary mode. this can keep a key good ways from the improvement sooner or later of up close and personal.

The present day system isn't having any faraway control leisure activity. depend upon others to work and No muscle withdrawal identifying and the proposed structure is having the mind wave examination for the sign which can be taken from the human cerebrum as appeared in the rectangular chart, is having controlling of the mechanical utilizing Human thoughts, Self controlled and working office for not to rely on others to work. This endeavor at Matlab, explains that tranquil speakme inside the sentiment of this stage is "discerning effort to express a word,

Revised Version Manuscript Received on July 10, 2019.

Dr.Bhukya Krishna, Professor, Dept. of CSE, CMR Technical Campus, Medchal, Hyderabad, Telangana, India

K. Ranjith Reddy, Assistant Professor, Dept. of CSE, CMR Technical Campus, Medchal, Hyderabad, Telangana, India portrayed through unpretentious advancements of inner talk organs without unquestionably voicing it." The way gets markers from the cerebrum to the muscle bunches while the client purposely vocalizes inside. The device later companions such banner with an out of entryways preparing framework. Like talk affirmation structures, it allows the customer to make solicitations to such gadgets, however without reporting anything.

Key Words: Brainwave sensor EEG, Wi-Fi, brainwave visualizer, Smart Sensors, Processors, Assistive Robot, Care givers

\section{INTRODUCTION}

In India, the more seasoned masses far and wide is tirelessly expanding. the amount of individuals 60 years old and extra snared expanded to directly around 900 million out of 2015 and resolved to achieve 2 billion by methods for 2050. In India, at present, the whole mean male and lady is fifty one million and 53 million. existing crisis facilities, care centers and unmistakable establishments starting at now convey care to severa physically disabled and more seasoned sufferers. these are over the zenith expensive and practical. more prominent master and sufferers might want to remain inside the comfort of their home wherein they feel extra sure than moving to any very estimated adult thought or human administrations work environments. in this manner, if more noteworthy mounted developed u.s.can complete self-care sports without every other person, it will encourage them to keep up self-governance and outfit them with a feeling of accomplishment and potential to acknowledge opportunity longer. The most extreme ideal strategy to help them is to give a real area that advances dynamic developing utilizing creative developments, for instance, Artificial Intelligence (AI), mind waves, brilliant homes and assistive robots. This mission recommendation outfits a self-care brandishing exercises course of action with a mind wave oversaw assistive robot, manufactured Intelligence and cloud organizations. the world changed over into new observations with imaginative endeavors and advances.

\section{TECHNOLOGIES}

\subsubsection{Artificial Intelligence}

programmed thinking is a procedure for making a $\mathrm{PC}$, a pc controlled automated, or an item think acutely, in the near way the savvy individuals suppose. AI is developed by pondering how human cerebrum thinks, and how individuals look at, choose, and work even as endeavoring to take care of a 
trouble, and after that creation utilization of the consequences of this test as a reason of making brilliant programming and systems.

\subsubsection{Internet of Things}

The net of elements is the device of real gadgets, vehicles, home machines, and various things embedded with equipment, programming, sensors, actuators, and system which enables these things to relate, amass and substitute insights, making open entryways for more noteworthy honest turning into an individual from of the physical global into PC basically based structures, bringing roughly profitability redesigns, financial favors, and diminished human endeavors

\subsubsection{Cloud computing}

dispensed figuring is shaping the computerized worldwide and creates as a key handling and organization arrange for sharing property including stages, programming bundles and the aggregate as organizations. regardless of the way that it brings our age unmatched figuring limit and fiscal advantages, the utilization of administered registering is up 'til now compelled at blessing inside the web as a result of the cloud organizations can simply live in cloud instead of our consistently ways of life condition. truth be exhorted, there are as however a masses of real capacity organized reachable administration demands that assigned processing should help insignificant on account of the "virtual obstruction".

\subsubsection{Android application}

The controlling contraption may be any android based cellphone/tab, etc having an android OS. The android controlling structure gives a top notch clever GUI that makes it simple for the buyer to control the framework. The transmitter utilizes an android application required for transmitting the insights. Our assignment proposes a wheelchair that can be cared for remotely through an android that permits getting and examining the rules. The wheelchair may also then be filled in as needed as told through the android programming

\subsection{System Architecture:}

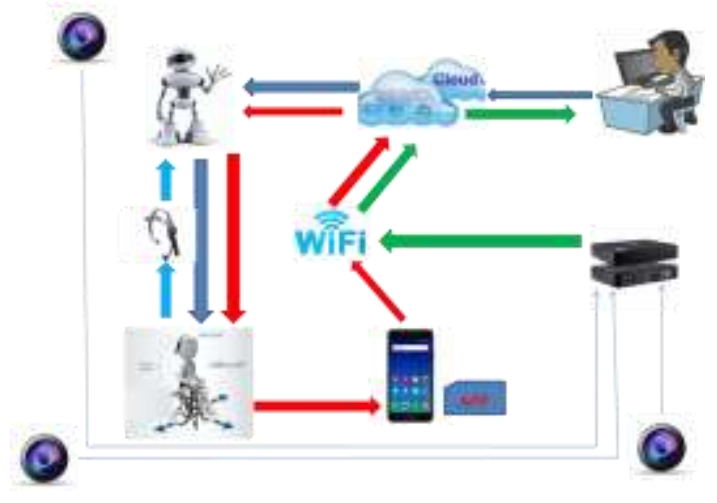

The central issue of our endeavor is essentially thought on real hindered and more prominent genius people in city zones. With the help of new advancement robot-AI, the weakened individuals can without a mess of a stretch make their lives loosened with comfort and faultlessness. This, yet what's more they can exit from mental weight for his or her disease. based gadget. The oversee gadget is composed with a cloud

they can realize their activities of themselves with no effort and support of others. we can watch it creates the impression that clearly from the plan, with the help of modify EGO gadget, mind experience gets each and every thought of injured and sends information to the robot. The cerebrum sensor is a worked in to change EGO gadget in head segment. for example, if the influenced individual thinks to have solutions, this data ship to the mechanical and performs genuine enthusiasm for the last outcomes. The robot gets the information with the guide of Bluetooth module. on the off hazard that mind feel fails to work ,, at that factor the robot gets certainties from android utility that is inbuilt to wheel-situate by WIFI through the cloud based absolutely server. The android utility plays out the brandishing exercises like vocal affirmation, remote oversee working. In the event that there must be an event of patient is out of gear mode, this system is orga-nized by chairman and audits to the mechanical through cloud. The executive is the person who shows the total structure is coming to viably.

\subsection{Methodologies:}

The fundamental methodology is quick joint effort among the considerations development of the customer and robot is perceived by utilizing Electro Encephalography (EEG) apparatus called "ALTER EGO" head-set. It conveys a terminal orchestrated in frontal capacity of the supporter's scalp. The assessing regards are recorded with the guide of the cathode through recognizing the electric movement of the considerations at a rate of 512 SPS (checks each second). The qualities recorded and later on transmitted through Bluetooth medium. EEG-ALGO writing computer programs is used to assess this EEG signal. certainties from change EGO musings Wave head set can be set away and later by applying sign embellishment technique we obtain the special cerebrum waves like alpha, beta, theta and delta. those sign are dismembered in EEG-ALGO programming. Dependent upon the thought, glint and consideration degree an appropriate computation is associated for perceiving the mechanical bearings. This procedure is direct providing pointers to automated by the cerebrum experience which controls patients considerations without an assistive.

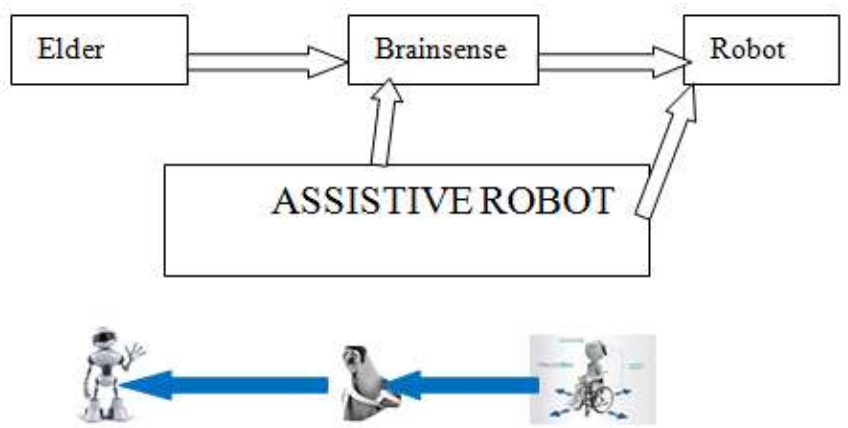

Second approach is if mind sense does not work appropriately, it prompts battery channel. All things considered we utilize portable application to control the robot. Patient can control the robot their very own and offer directions to the robot by means of WIFI module through the cloud information base. 

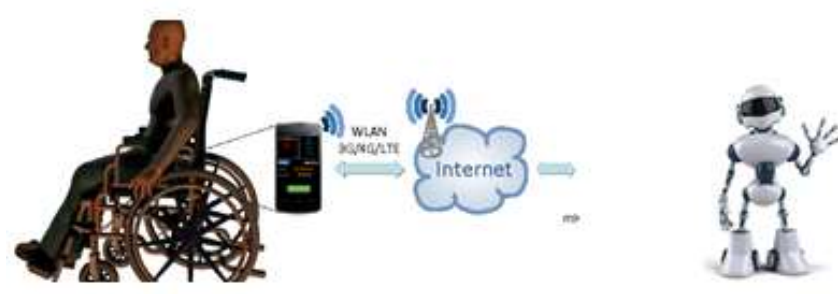

\subsection{Peripheral Description}

Brainwave Headset which is given with the guide of modify EGO advancement and individuals sign can be traveled through utilizing Bluetooth that is there inside the contemplations wave headset, for this considerations wave headset need to exhibit power utilizing an AAA battery. The musings wave headset goes with quality switch, a sensor tip, versatile ear arm and a ground alliance ear with a mouthpiece that stretches out over the wonderful to just underneath the lip. on this Headset they use Non-meddling sensor that may not make any torment the client who had been the headset. alongside embeddings an AAA battery turn on the mind wave headset utilizing the vitality switch the LED pointer will on and if the pink concealing mellow now not on circumstance the headset is overseen not on and not related with the mechanical Bluetooth. at the off hazard that the Blue concealing now not squinting that recommends the headset is energized on and related. on the off hazard that the red or blue concealing flashes fastly it exhibits that the Battery getting low. data transmitted by method for the considerations wave headset can be gotten by means of the automated Bluetooth authority. After the exploration of this records, this data might be sent to the robot module using successive information transmission for instance using Bluetooth module. In that robot module there might be a Bluetooth recipient gets the records that is transmitted by methods for the Bluetooth transmitter. As showed by means of the data gotten by utilizing the Bluetooth the ARM processor will convey the course to the robot that are identified with an exchange and an intention power circuit.

\subsubsection{Alter-Ego}

The objectives of change Ego are to mentally development individuals, interchange the way wherein individuals talk with each extraordinary, and engage a reasonable gateway to mechanized realities (organizations and bundles) wherein the affiliation is trademark in inclination to something outward. Our present interfaces are a limit to simple and private human-gadget correspondence. individuals either need to move their consideration a long way from their condition to kind, or they have to nation their non-open messages for all to hear, out in the open with the BRAIN COMPUTER IN-TERFACE(BCI). direct Ego overcomes these obstacles by methods for empowering customers to unobtrusively and continually interface with a PC without the necessity for unequivocal exercises. It engages a strategy for human-PC association without dissuading the buyer's average acknowledgment, thusly giving the customer a hazard to remain present in her condition. The alter Ego headset gets the neuromuscular sign that happen when individuals intend to talk. It by then uses a neural framework to imitate the word.
The structure can examine the ones facial sign with ninety two percent precision. The headset is worn on one ear with a mouthpiece that stretches out over the excellent to under the lip. The earpiece comprises of various bone-conduction headphones that transmit vibrations through the issue that stays to be worked out internal ear. The modify Ego can be used for controlling IOT contraptions, virtual-and expanded reality applications.

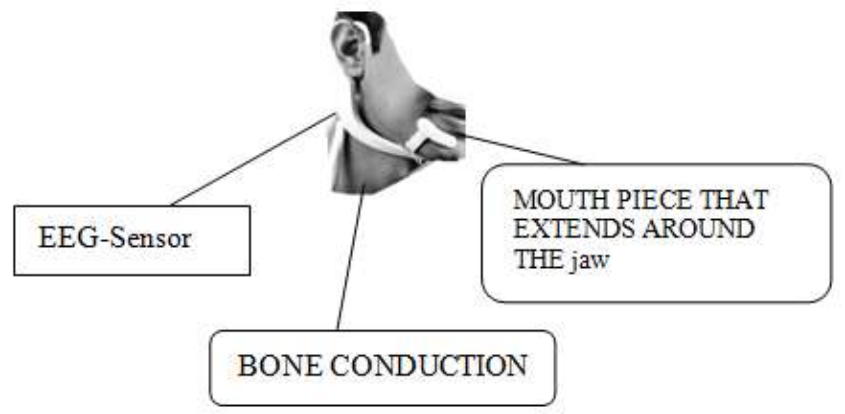

\subsection{Principle of $B C I$}

A BCI has an input (e.g. electrophysiological activity from the user), an output (i.e. device commands), segments that make an interpretation of contribution to yield and a conference that comes to a decision the onset,offset, and timing ofoperation. signal from the cerebrum are procured by means of terminals at the scalp or in the head and prepared to separate explicit sign highlights (as an instance amplitudes of evoked opportunities or tangible engine cortex rhythms, terminating fees of cortical neurons) that replicate the consumer's aim. those highlights are converted into guidelines that work a gadget (as an example a basic phrase dealing with application, a wheelchair, or a neuroprosthesis).achievement of BCI challenge relies upon the communication of versatile controllers, customer and framework. The patron have to create and preserve up high-quality connection between's his or her motive and the signal highlights used by the BCI and the BCI have to pick and pay attention consists of that the consumer can control and must make an interpretation of these highlights into system instructions appropriately and successfully.

\section{SENSOR DESCRIPTION}

It is an efficient means it has a tendency to divide brain-controlled mobile robots into 2 classes consistent with their operational modes. One class is termed "direct management by the BCI," which implies that the BCI unravels graphical report signals into development headings to control robots clearly United nations work environment recently built up a mind controlled mechanical seat whose left or appropriate turning inclinations are genuinely restricted by utilizing assessing movement rules translated from shopper cerebrum pointers simultaneously as imagining left or legitimate extremity drifts, and attempted this methodology in evident things. The mechanical level is demonstrated in like manner connected a BCI reliant on motor imagery to amass a cerebrum oversaw transportable automated, as depicted

Published By: 


\section{NETWORK SENSOR FOR BRAIN WAVE WITH AUTOMATED ROBOT ASSISTANCE}

which may also perform 3 development directions which is squint, reflected picture and consideration all in all with turning left and right and going ahead of time, and broad this gadget all through a planet.

\section{TESTING \& RESULTS}

Accuracy and safety are the most important part of this project. In order to test the accuracy of the robot the following methods are used, and each method will be tested for three rounds.

During the test, the number of correct command means the motion of the robot match the command sent by the user; the number of the wrong command means either: 1) The motion does not match the command. 2) The command is seen as noise. 3) Noise is seen as a command. The first test is focused on the autonomous terrain detection (see Figure 1). During the testing, the robot should detect the terrain and avoid falling from the stair. The testing criteria are to count the times of falling from the stair. In this test, a higher-level ground will be used to simulate the stair.

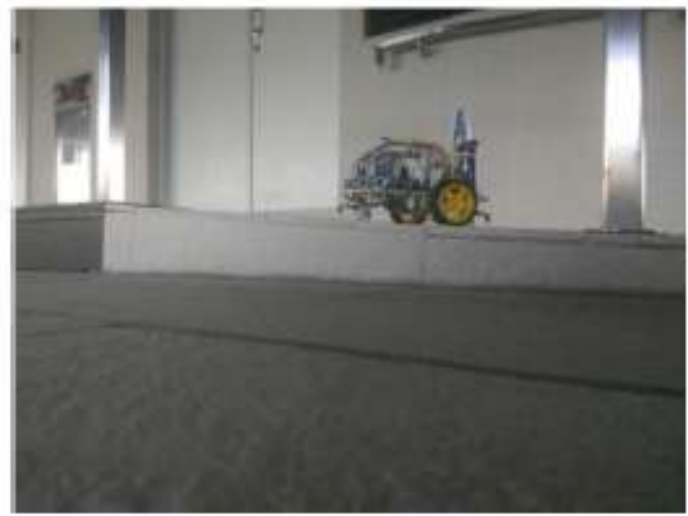

Figure 1. Autonomous terrain detection

The testing results are listed in Table 1.

\begin{tabular}{llll} 
Table 1. Testing results of autonomous terrain detection \\
\hline \multirow{2}{*}{ Round } & $\begin{array}{l}\text { Number of times trying to } \\
\text { fall from the bigh-level } \\
\text { ground }\end{array}$ & Results & Accuracy \\
\hline 1 & 2 & Boch avoided & $100 \%$ \\
2 & 2 & Boch avoided & $100 \%$ \\
3 & 2 & Boch avoided & $100 \%$ \\
\hline
\end{tabular}

During the test, the robot can avoid falling from stair successfully. When the sensors detected the distance between the robot body and the ground is too large, it will go back and turn to avoid falling from stairs.

The next test is based on a simple rectangular map (see Figure 2). During the testing, the robot should follow the rectangle drawn on the floor. The testing criteria are to count the times of incorrect command received. In this test, 5 checkpoints are labeled on the ground. The robot should reach each checkpoint in the order of: red, orange, yellow, green, and finally stop at blue.

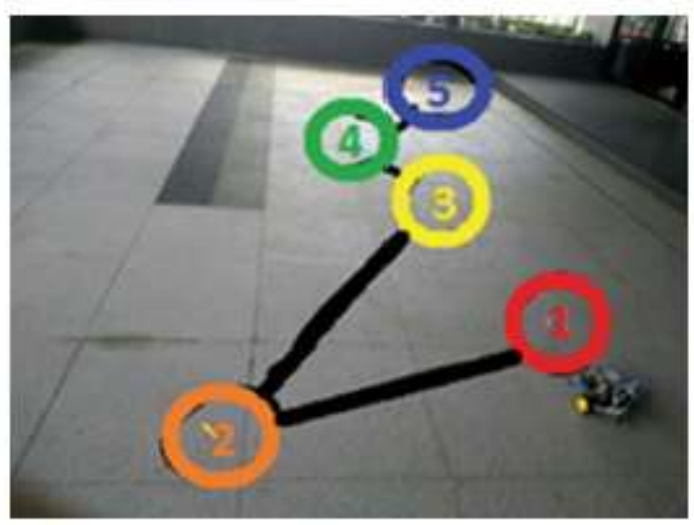

Figure 2. Test on a simple map

The testing results are listed in Table 2.

Table 2. Testing results of running on a simple map

\begin{tabular}{lllll}
\hline Round & $\begin{array}{l}\text { The number of } \\
\text { commands sent }\end{array}$ & Correct & Wrong & Accuracy \\
\hline 1 & 20 & 18 & 2 & $90 \%$ \\
2 & 19 & 18 & 1 & $94.7 \%$ \\
3 & 15 & 15 & 0 & $100 \%$ \\
\hline
\end{tabular}

During the test, the robot can pass through all the checkpoints successfully. Even sometime the robot may not recognize the user's command correctly, the user can send the command again to avoid the robot being derailed or directly stop the robot by blinking three times and more rapid. The stop command is the most sensitive and accurate command so that it can use to prevent the risk happen.

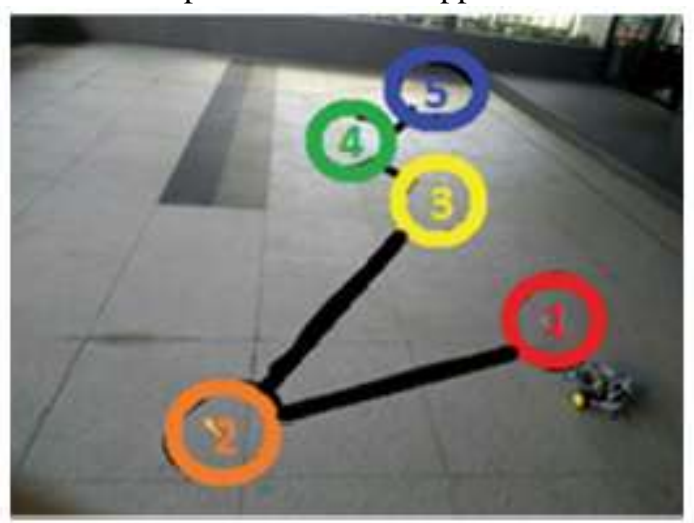

Figure 3. Test on an irregular map

The next test is based on an irregular map (see Figure 3) During the testing, the robot should follow the irregular path on the floor. The testing criteria is to count the times of incorrect command received. In this test, 5 checkpoints are labeled on the ground. The robot should reach each checkpoint in the order of: red, orange, yellow, green, and finally stop at blue.

The testing results are listed in Table 3.

\begin{tabular}{lllll} 
Table 3. Testing results of running on an irregular map \\
Round & $\begin{array}{c}\text { The number of } \\
\text { commands sent }\end{array}$ & Correct & Wrong & Accuracy \\
\hline 1 & 22 & 20 & 2 & $90.9 \%$ \\
2 & 26 & 23 & 3 & $88.4 \%$ \\
3 & 20 & 18 & 2 & $90 \%$ \\
\hline
\end{tabular}




\section{CONCLUSION}

The sign delivered by method for mind transformed into gotten by means of the cerebrum sensor and it will segment into groups and the package insights transmitted to far away medium. Level analyzer unit (LAU) will get the brainwave rough realities and it'll focus and process the sign using Matlab arrange. At that factor the pointers will send the house work environment the module. The endeavor worked with human musings assumption and the on off kingdom of home contraption depends upon on changing the muscle improvement with flashing.. By then the oversee rules could be transmitted to the programmed module to way. With this total system, we will stream a robot as indicated by the human concerns and it tends to be wound up through gleam muscle pressure. The advanced works of art of psyche oversaw adaptable robots have gotten a great deal of thought given that they can help take compactness lower back to people with destroying neuro-solid issue and along those follows improve their non-open fulfillment. improving the BCI structure execution to make brain oversaw flexible robots usable in obvious conditions.

\section{ACKNOWLEDGEMENT}

The authors would like to thanks to Chairman and Director of CMR Technical Campus for valuable suggestions and also express sincere gratitude to the organization for their constant encouragement and co-operation

\section{REFERENCES}

1. Cho JH, Chang SA, Kwon HS, Choi YH, KoSH, Moon SD, Yoo SJ, Song KH, Son HS, Kim HS, Lee WC, Cha BY, Son HY \& Yoon KH (2006), Long-term effect of the internet-based glucose monitoring system on HbA1c Reduction and glucose stability: a 30-month follow-up study for diabetes management with a ubiquitous medical care system. Diabetes Care 29, 2625-2631.

2. Fauci AS, Braunwald E, Kasper DL \& Hauser SL (2008), Principles of Harrison's Internal Medicine, Vol. 9, 17thedn. McGraw-Hill, New York, NY, pp.2275-2304.

3. Kim HS \& Jeong HS (2007), A nurse short message service by cellular phone in type- 2 diabetic patients for six months. Journal of Clinical Nursing 16, 1082-1087.

4. Lee JR, Kim SA, Yoo JW \& Kang YK (2007), The present status of diabetes education and the role recognition as a diabetes educator of nurses in korea. Diabetes Research and Clinical Practice 77, 199-204.

5. McMahon GT, Gomes HE, Hohne SH, Hu TM, Levine BA \& Conlin PR (2005), Web-based care management in patients with poorly controlled diabetes. Diabetes Care 28, 1624-1629.

6. Thakurdesai PA, Kole PL \& Pareek RP (2004), Evaluation of the quality and contents of diabetes mellitus patient education on Internet. Patient Education and Counseling 53, 309-313.

7. Dany Bright, Amrita Nair, Devashish Salvekar and Prof.Swati Bhiskar,"EEG-Based Brain Controlled Prosthetic Arm", Pune,Jun 9-11,2016.

a. Luzheng Bi, Xin-An Fan, Yili Liu, "EEG-Based BrainControlled Mobile Robots: A Survey “, IEEE transaction on human machine systems", vol. 43, March 2013, pp. 161-176.

8. J Butterfass, G Hirzinger, S Knoch - Robotics and Automation, 1998 - ieeexplore.ieee.org 